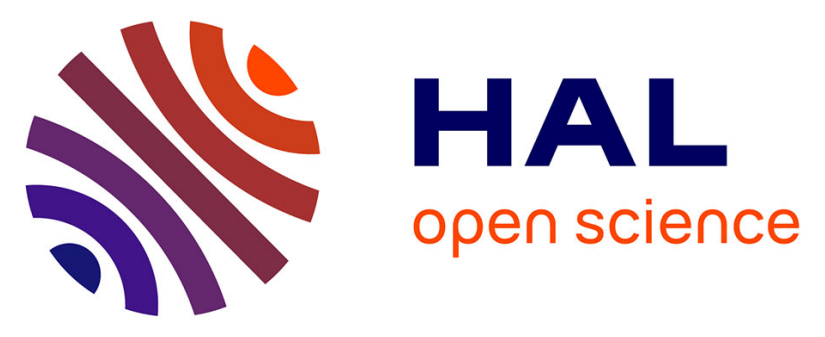

\title{
Photonic-crystal GaN light-emitting diodes with tailored guided modes distribution
}

\author{
Aurélien David, Tetsuo Fujii, Rajat Sharma, Kelly Mcgroddy, Shuji \\ Nakamura, Steven P. Denbaars, Evelyn L. Hu, Claude Weisbuch, Henri \\ Benisty
}

\section{To cite this version:}

Aurélien David, Tetsuo Fujii, Rajat Sharma, Kelly Mcgroddy, Shuji Nakamura, et al.. Photoniccrystal GaN light-emitting diodes with tailored guided modes distribution. Applied Physics Letters, 2006, 88 (6), pp.061124. 10.1063/1.2171475 . hal-00869409

\section{HAL Id: hal-00869409 \\ https://hal-iogs.archives-ouvertes.fr/hal-00869409}

Submitted on 3 Oct 2013

HAL is a multi-disciplinary open access archive for the deposit and dissemination of scientific research documents, whether they are published or not. The documents may come from teaching and research institutions in France or abroad, or from public or private research centers.
L'archive ouverte pluridisciplinaire HAL, est destinée au dépôt et à la diffusion de documents scientifiques de niveau recherche, publiés ou non, émanant des établissements d'enseignement et de recherche français ou étrangers, des laboratoires publics ou privés. 


\title{
Photonic-crystal GaN light-emitting diodes with tailored guided modes distribution
}

\author{
Aurélien David, ${ }^{\text {a) }}$ Tetsuo Fujii, ${ }^{\text {b) }}$ Rajat Sharma, Kelly McGroddy, Shuji Nakamura, \\ Steven P. DenBaars, Evelyn L. Hu, and Claude Weisbuch \\ Materials Department, University of California, Santa Barbara, California 93106 \\ Henri Benisty \\ Laboratoire Charles Fabry de l'Institut d'Optique, Bat 50391403 Orsay, France
}

(Received 15 August 2005; accepted 13 December 2005; published online 10 February 2006)

\begin{abstract}
We relate the currently limited efficiency of photonic crystal (PhC)-assisted gallium nitride light-emitting diodes (LEDs) to the existence of unextracted guided modes. To remedy this, we introduce epitaxial structures which modify the distribution of guided modes. LEDs are fabricated according to this concept, and the tailored band structure is determined experimentally. We investigate theoretically the consequences of this improvement, which significantly enhances the potential for efficient light extraction by PhCs. () 2006 American Institute of Physics.
\end{abstract}

[DOI: $10.1063 / 1.2171475$ ]

Gallium nitride $(\mathrm{GaN})$ based materials, which emit in the $\mathrm{UV}$ and blue-green wavelengths, represent a promising but challenging solution towards efficient solid-state lighting. Conventional GaN-based light-emitting diodes (LEDs) still suffer from limited light extraction, due to total internal refraction (TIR) of the emitted light at the interface between semiconductor and air. Efficient solutions, such as flip-chip ${ }^{1}$ or surface-roughened ${ }^{2}$ LEDs, unfortunately require complex fabrication procedures. On the other hand, photonic crystal $(\mathrm{PhC})$ were studied extensively in a variety of materials in the last decade, as an efficient means to enhance light extraction in LEDs, while keeping a full planar device fabrication. More recently, another stategy was also explored: light extraction by coupling to the surface plasmons of Ag films. ${ }^{3-5}$

Two schemes can be considered for PhC-assisted LEDs: formation of band gaps to prevent emission into guided modes, ${ }^{6}$ or PhCs acting as diffraction gratings to extract guided light. ${ }^{7-11}$ While good results have been reported regarding the former scheme for low-temperature photoluminescence experiments, ${ }^{12}$ extension to room temperature may be limited by increased loss due to nonradiative recombination. Hence, we focus on the second scheme, using PhCs as diffraction gratings for guided modes.

Several recent approaches have incorporated $\mathrm{PhC}$ structures in GaN-based LEDs, leading to enhancements of the light extraction efficiency by factors of $\times 1.5-\times 1.6$ for blue LEDs. ${ }^{13,14}$ As it is known that only $\sim 10 \%$ of the total emitted light is directly radiated in air through direct emission (top and bottom) in an unmounted GaN structure, it is reasonable to hope for PhC-LEDs with far larger efficiencies than those reported up to now.

So far, however, no evaluation has been made of the full potential of PhC-assisted light extraction in GaN-based LEDs. In particular, there is a lack of clarity on the exact action of the $\mathrm{PhC}$ structure, e.g., which fraction of the emitted light it is expected to extract. Of course, basic arguments can be borrowed from previous work on other materials, such as GaAs. ${ }^{8,10,15}$ Yet in view of the specificities of GaN

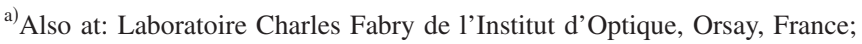
electronic mail: aurelien.david@polytechnique.org

${ }^{b)}$ Also at: NICP/ERATO UCSB.
}

(layers thickness, transparent substrate, low refractive index...), it seems worthwhile to re-explore the concept of light extraction assisted by $\mathrm{PhC}$ gratings in this material.

In this letter, we address this issue, point out some limitations in usual PhC structures, and suggest a straightforward way of improvement by tailoring the structure of guided modes in the epitaxial layers.

Let us first consider the various paths accessible to light emitted in a classic GaN LED (Fig. 1): $6 \%$ of the light is emitted in air towards the top (assuming a transparent $p$ contact), which can be doubled if a mirror is placed under the sapphire substrate. $22 \%$ is trapped by TIR but can propagate in the sapphire-we call this fraction the substrate light. Finally, $66 \%$ is trapped in the GaN layer by TIR at the air and sapphire interfaces. We label this light as guided light.

Substrate light has negligible interaction with the $\mathrm{PhC}$ region, as it propagates for long distances in the thick $(\sim 500 \mu \mathrm{m})$ transparent sapphire wafer, and impinges on the $\mathrm{PhC}$ no more than once before exiting the LED region. Therefore, the $\mathrm{PhC}$ only aims at extracting guided light.

We showed recently that, even though for good material quality the GaN layer needs to be thick $(\sim 3-5 \mu \mathrm{m})$, it can still be studied as a (strongly multimode) waveguide,
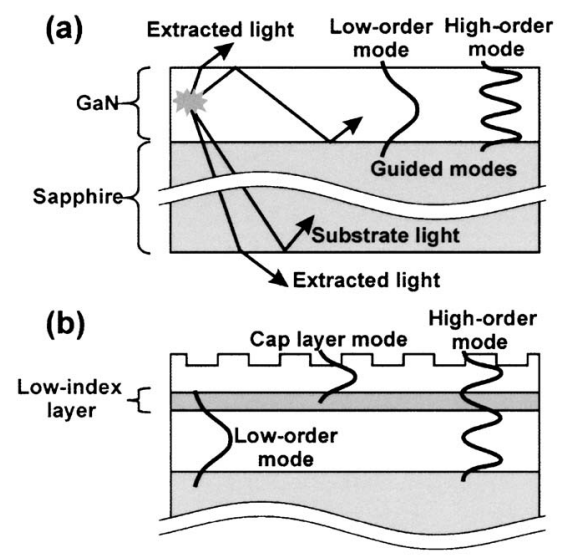

FIG. 1. (a) Possible paths for emitted light in a GaN structure. (b) Various guided modes in presence of a confining low-index layer. 

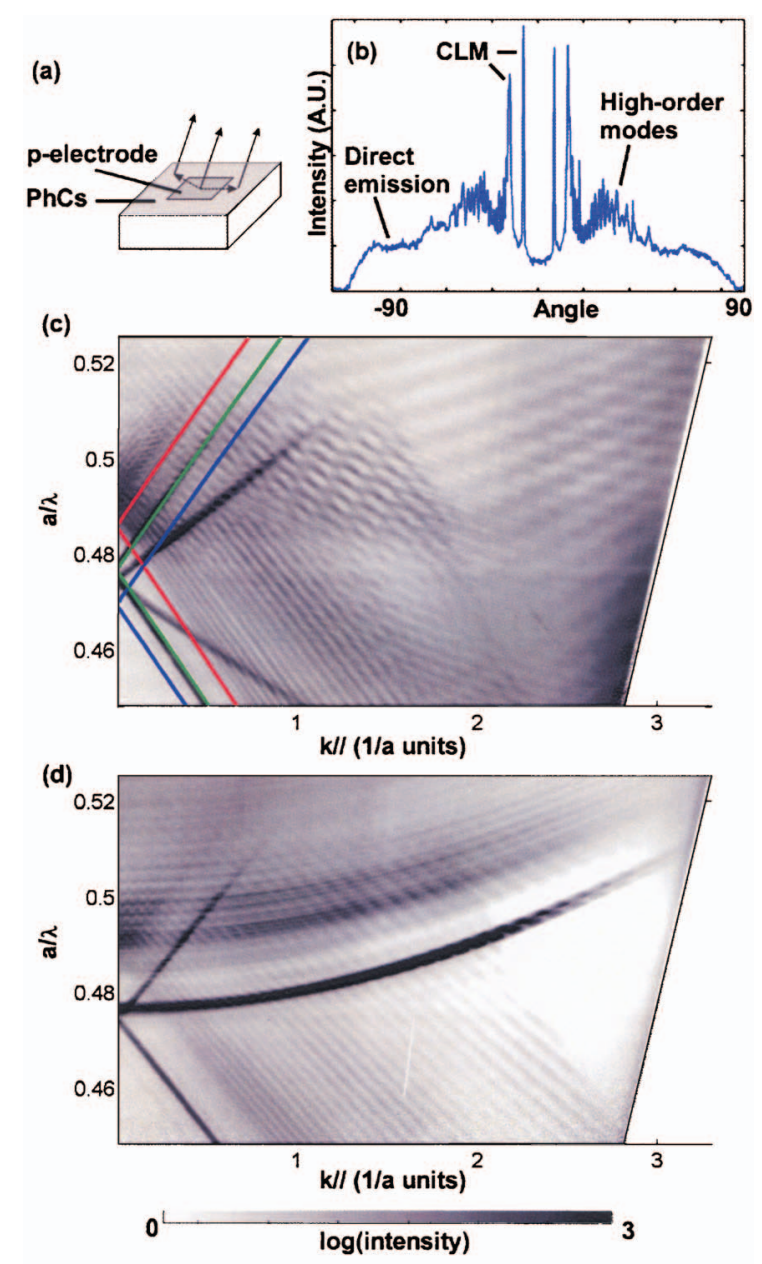

FIG. 2. (Color) (a) Geometry of an LED. (b) Angular spectrum at $\lambda$ $=470 \mathrm{~nm}$. (c) Band structure of the LED along the $\Gamma \mathrm{M}$ direction of the PhC (log scale), light collected in TE polarization. The strongest bands correspond to the CLM, other bands to the high order modes. The blue and red lines correspond to the lightlines of $\mathrm{GaN}$ and $\mathrm{AlGaN}$. The green line fits a guided mode with effective index $n_{\text {eff }}=n_{\mathrm{GaN}}-0.045$. (d) Same as (c), $\Gamma K$ direction and TM polarization.

wherein the diffraction efficiency can be heavily dependent on the mode. ${ }^{16}$ If we label modes by their effective index $n_{\mathrm{eff}}$, and $n_{\mathrm{GaN}}$ is the index of GaN, low-order modes (say, $\left.n_{\mathrm{GaN}}>n_{\mathrm{eff}}>n_{\mathrm{GaN}}-0.15\right)$ are mostly localized in the core of the $\mathrm{GaN}$ waveguide so that they barely overlap with the $\mathrm{PhC}$, leading to poor diffraction efficiency. On the other hand, high-order modes $\left(n_{\mathrm{GaN}}-0.15>n_{\mathrm{eff}}>n_{\text {sapphire }}\right)$ have increased overlap with the $\mathrm{PhC}$ and acquire a better extraction rate. Unfortunately, low-order modes also carry a large portion of the total light emission, on the order of $30 \%$. $^{.7}$

In our first experiments, using "regular" shallow $\mathrm{PhCs}$ $(\sim \lambda / n)$ on top of a thick GaN layer, diffraction of these modes could not be observed, a fact we attributed to their poor overlap with the PhC: the corresponding bands did not appear in angular-resolved photoluminescence measurements. One could therefore consider etching the $\mathrm{PhC}$ deeper $(\sim 1 \mu \mathrm{m})$ in order to increase the interaction between the $\mathrm{PhC}$ and these modes. However, as the average index in the $\mathrm{PhC}$ region (typically $<2$ ) is smaller than the effective index of the low-order modes, they are strongly evanescent in the etched region so that their diffraction does not increase significantly for a PhC depth larger than $\sim \lambda / n$.

Therefore, our approach consists in modifying the distribution of guided modes while maintaining a shallow PhC.

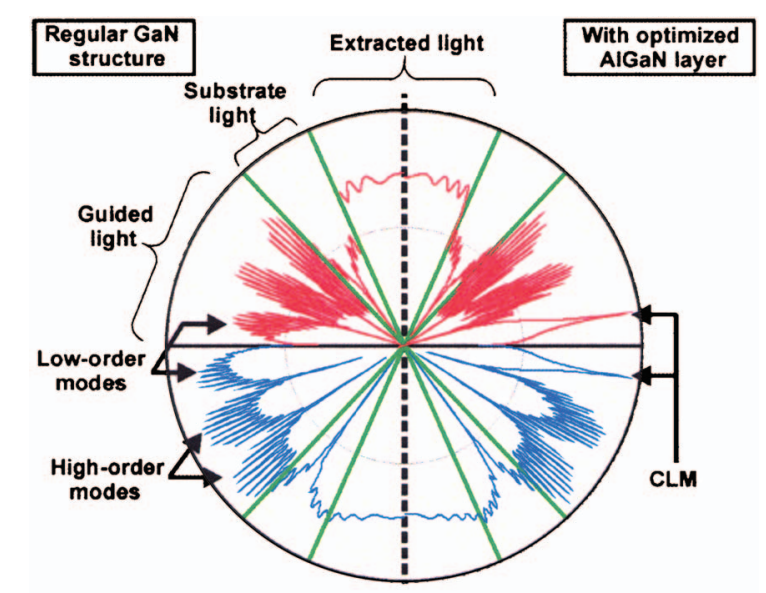

FIG. 3. (Color) Emission of a dipole plane in a GaN structure as a function of the polar angle of emission (log scale, TE polarization, red=upwards, blue $=$ downwards). The lightlines of air and sapphire are depicted by green lines. Left half: typical GaN structure, 30\% of light is emitted in low-order modes and not coupled to the PhC. Right half: same structure with an AlGaN layer, the low-order modes are replaced by a CLM carrying $30 \%$ of the total light.

High-order modes need not be altered, but one has to modify low-order modes. To this effect, let us consider a structure formed of a GaN buffer, a lower-index layer-for instance an $\mathrm{Al}_{x} \mathrm{Ga}_{1-x} \mathrm{~N}$ layer-and a GaN cap layer containing the $p-n$ junction and active InGaN quantum wells $(\mathrm{QW})$ region. The modes supported by the new structure are depicted in Fig. 1(b). Of course, the former low-order modes localized in the buffer still exist, but very little light is emitted into these modes due to their poor overlap with the $\mathrm{QW}$ region. On the other hand, if the GaN cap layer on top of the $\mathrm{AlGaN}$ is thick enough, it supports a mode of high $n_{\text {eff }}\left(n_{\mathrm{GaN}}>n_{\mathrm{eff}}\right.$ $\left.>n_{\mathrm{AlGaN}}\right)$. This cap layer mode (CLM) receives most of the light emitted at glancing angles as it is intense in the QW region, and interacts strongly with the $\mathrm{PhC}$, with which it has a good vertical overlap. Finally, high-order $\left(n_{\text {eff }}<n_{\text {AlGaN }}\right)$ modes are hardly affected by this slight change of index.

This approach is supported by a frequency-domain three-dimensional modeling of the $\mathrm{PhC}$ structure, which gives access to the extraction length $L_{\text {decay }}$ for each mode (characterizing the exponential decay of modes along propagation in the $\mathrm{PhC}$ ). We obtain, respectively, for the ground state mode (lowest-order mode), the CLM, and a typical high-order mode: $L_{\text {ground }} \sim \infty, L_{\mathrm{CLM}} \sim 100 \mu \mathrm{m}, L_{\text {high-order }}$ $\sim 60-200 \mu \mathrm{m}$ depending on the mode and frequency.

Such a structure was grown by metalorganic chemical vapor deposition. The epitaxial structure consisted of a 3- $\mu$ m-thick GaN buffer, a 800-nm-thick $\mathrm{Al}_{0.1} \mathrm{Ga}_{0.9} \mathrm{~N}$ layer, and a 600-nm-thick GaN cap layer with a multi-QW region embedded at its center. LEDs were formed on the material, with a $p$-contact injection area of $100 \times 100 \mu \mathrm{m}$. The area surrounding the $p$ contact was patterned with photonic crystals using electron-beam lithography and a $\mathrm{SiO}_{2}$ hard mask. The $\mathrm{PhCs}$ were then etched by reactive ion etching, to a depth of $\sim 250 \mathrm{~nm}$. The index contrast provided by the AlGaN layer is about $0.1{ }^{18}$ sufficient to isolate the CLM from the buffer considering the thickness of the AlGaN layer.

To access the band structure of the LEDs, we used angular-resolved measurements as in our previous work. ${ }^{16}$ Electroluminescence was collected at all angles $\left(-90^{\circ}\right.$ to $90^{\circ}$ ) by a rotating arm and analyzed in a spectrometer. We 
collected both the direct emission of the LED, and the diffraction of the surrounding $\mathrm{PhC}$. Collection angles were then converted to in-plane wave vectors $\mathbf{k}_{\|}$and wavelengths to frequencies $a / \lambda$, yielding the band structure (Fig. 2). In contrast to previous measurements, ${ }^{16}$ the direct emission from the LED could not be isolated and subtracted here, since the $\mathrm{PhC}$ diffracted in all directions. Therefore, the direct emission is present as a gray background (modulated by FabryPérot interferences of the GaN layer) on the spectra of Fig. 2.

On the sample presented here, the lattice constant is $a$ $=215 \mathrm{~nm}$ so that emission is tuned around the second Bragg order of the PhC. ${ }^{9}$ The filling factor is $f=0.3$. As in previously measured PhCs samples, ${ }^{16}$ we observe sets of bands stemming from the high-order modes. However, in contrast to previous results, a new, intense band now appears at lower frequency. This band corresponds to the CLM. A simple fit by a line of dispersion $\omega=k c / n_{\text {eff }}$ [green line on Fig. 2(c)] yields the effective index of this mode: $n_{\mathrm{eff}}=n_{\mathrm{GaN}}-0.045$, midway between the indices of $\mathrm{GaN}$ and $\mathrm{AlGaN}$ layer, confirming that it is guided in the cap layer. The intensity of this mode - notice the log scale of Figs. 2(c) and 2(d) -is evidence of the large fraction of light it receives from the QWs.

In order to measure the extraction efficiency of the LEDs, we placed a mirror under the sapphire layer, thus mimicking a realistic device where light extracted downwards is redirected upwards. We compared the PhC-LEDs to classic LEDs (simultaneously formed on the same wafer with no surrounding $\mathrm{PhCs}$ ). The extraction enhancement, measured by a photodiode with a numerical aperture 0.5 , was only $+70 \%$ at an injection current of $10 \mathrm{~mA} \mathrm{dc}$ $\left(100 \mathrm{~A} / \mathrm{cm}^{2}\right)$. This modest enhancement is not surprising owing to the lack of optimization in these LEDs. Indeed, in our epitaxial layers, the placement of the QWs with respect to the AlGaN layer is so far not optimized. Moreover, in these test structures, the length of the $\mathrm{PhC}$ surrounding the injection area is only $80 \mu \mathrm{m}$, clearly not sufficient to extract all the guided light. It is also possible to extrapolate the extraction enhancement from angular measurements, but this should be carried out with care as light emission is quite dependent on the azimuthal angle and polarization of collected light - see the difference between band structures of Figs. 2(c) and 2(d). By carefully fitting the angular measurements with a simple ray-tracing model for extraction, we deduced an extraction enhancement of $+100 \%$. From these two figures, we estimate the total light extraction efficiency to be between $20 \%$ and $24 \%$. It should also be noted that this is obtained at no compromise of the $I-V$ characteristics of the LEDs, which are similar for patterned and unpatterned LEDs, mainly because the contact area is free of patterning.

To estimate further the potential of our approach, we calculated the QW radiation pattern in our structure, using the transfer matrix method with a dipole source. ${ }^{17}$ It turns out that if a QW is located at the antinode of the CLM, coupling between the QW and the CLM is maximal. In an optimized structure (consisting of a 300-nm-thick cap layer with a QW placed in its middle, and a $1-\mu$ m-thick $\mathrm{Al}_{0.1} \mathrm{Ga}_{0.9} \mathrm{~N}$ layer) this mode can receive up to $30 \%$ of the total emission of the $\mathrm{QW}$, even though the index contrast between $\mathrm{GaN}$ and $\mathrm{AlGaN}$ is only 0.1 . Figure 3 depicts the dipole emission pattern without $\mathrm{AlGaN}$ and with the optimized $\mathrm{AlGaN}$ layer. In the optimized structure, nearly no light is emitted in the loworder modes. Only the CLM and the high-order modes carry light, so that practically all of the guided light is prone to interaction with the $\mathrm{PhC}$.

At this point, the limitation in $\mathrm{PhC}$ extraction is no longer governed by the "vertical" direction, but by in-plane phenomena. One is the azimuthal anisotropy of the $\mathrm{PhC}$ (e.g., which crystal directions can extract light) and another is parasitic diffraction to the substrate, as additional diffraction orders can couple guided light to substrate light. ${ }^{8,11} \mathrm{We}$ believe that future efforts addressing these points are the path to ultraefficient PhC LEDs.

In conclusion, we have clarified the purpose of $\mathrm{PhC}$ diffraction gratings in extracting light in GaN-based LEDs. After pointing out some issues due to the multiple modes in thick GaN layers, we have studied and characterized a solution based on tailoring the structure of guided modes, which enables nearly all of the guided light to be prone to interaction with the PhC. The fabricated LEDs, while still far from an optimal design, confirm the validity of the approach of modes tailoring. This improvement comes at no cost or additional fabrication steps, and could easily be combined with future optimization of the intrinsic $\mathrm{PhC}$ properties, bringing us closer to ultraefficient PhC-assisted LEDs.

This work was supported by the Solid State Light and Display Center at UCSB, and the Department of Energy.

${ }^{1}$ J. J. Wierer, D. A. Steigerwald, M. R. Krames, J. J. O'Shea, M. J. Ludowise, G. Christenson, Y.-C. Shen, C. Lowery, P. S. Martin, S. Subramanya W. Gotz, N. F. Gardner, R. S. Kern, S. A. Stockman, Appl. Phys. Lett. 78, 3379 (2001).

${ }^{2}$ T. Fujii, Y. Gao, R. Sharma, E. L. Hu, S. P. DenBaars, and S. Nakamura, Appl. Phys. Lett. 84, 855 (2004).

${ }^{3}$ I. Gontijo, M. Boroditsky, E. Yablonovitch, S. Keller, U. K. Mishra, and S. P. DenBaars, Phys. Rev. B 60, 11564 (1999).

${ }^{4}$ K. Okamoto, I. Niki, A. Shvartser, Y. Narukawa, T. Mukai, and A. Scherer, Nat. Mater. 3, 601 (2004).

${ }^{5}$ K. Okamoto, I. Niki, A. Scherer, Y. Narukawa, T. Mukai, and Y. Kawakami, Appl. Phys. Lett. 87, 071102 (2005).

${ }^{6}$ S. Fan, P. R. Villeneuve, J. D. Joannopoulos, and E. F. Schubert, Phys. Rev. Lett. 78, 3294 (1997).

${ }^{7}$ M. Boroditsky, T. F. Krauss, R. Coccioli, R. Vrijen, R. Bhat, and E. Yablonovitch, Appl. Phys. Lett. 75, 1036 (1999).

${ }^{8}$ M. Rattier, H. Benisty, E. Schwoob, C. Weisbuch, T. F. Krauss, C. J. M. Smith, R. Houdre, and U. Oesterle, Appl. Phys. Lett. 83, 1283 (2003).

${ }^{9}$ N. Eriksson, M. Hagberg, and A. Larsson, IEEE J. Quantum Electron. 32, 1038 (1996).

${ }^{10}$ D. Delbeke, P. Bienstman, R. Bockstaele, and R. Baets, J. Opt. Soc. Am. B 19, 871 (2002).

${ }^{11}$ A.-L. Fehrembach, S. Enoch, and A. Sentenac, Appl. Phys. Lett. 79, 4280 (2001).

${ }^{12}$ M. Fujita, S. Takahashi, Y. Tanaka, T. Asano, and S. Noda, Science 308, 1296 (2005).

${ }^{13}$ T. N. Oder, J. Shakya, J. Y. Lin, and H. X. Jiang, Appl. Phys. Lett. 83, 1231 (2003).

${ }^{14}$ J. J. Wierer, M. R. Krames, J. E. Epler, N. F. Gardner, M. G. Craford, J. R. Wendt, J. A. Simmons, and M. M. Sigalas, Appl. Phys. Lett. 84, 3885 (2004).

${ }^{15}$ M. Rattier, H. Benisty, R. P. Stanley, J.-F. Carlin, R. Houdré, U. Oesterle, C. J. M. Smith, C. Weisbuch, and T. F. Krauss, IEEE J. Sel. Top. Quantum Electron. 8, 238 (2002).

${ }^{16}$ A. David, C. Meier, R. Sharma, F. S. Diana, S. P. DenBaars, E. Hu, S. Nakamura, C. Weisbuch, and H. Benisty, Appl. Phys. Lett. 87, 101107 (2005).

${ }^{17}$ H. Benisty, H. D. Neve, and C. Weisbuch, IEEE J. Quantum Electron. 34, 1612, 1632 (1998).

${ }^{18}$ The index dispersion of GaN is given by Ref. 19, and the index of AlGaN is assumed to follow the dispersion of GaN, shifted by the change in band gap.

${ }^{19}$ H. Y. Zhang, X. H. He, Y. H. Shih, M. Schurman, Z. C. Feng, and R. A. Stall, Opt. Lett. 21, 1529 (1996). 